\title{
TOPICS IN NEUROLOGY
}

\section{Thoracic Schwannoma in an Adult Male Presenting With Thoracic Pain: A Case Report}

\author{
Andrew M. Rodgers, DC, ${ }^{a}$ and Kim B. Khauv, DC, MPH ${ }^{\mathrm{b}}$
}

\begin{abstract}
Objective: The purpose of this case report was to describe a patient presenting with back pain subsequently diagnosed with thoracic schwannoma.

Clinical Features: A 45-year-old male presented to a chiropractic practice with mid-back, low back, and sacroiliac pain. Orthopedic examination revealed joint dysfunction in the thoracic-sacroiliac region with normal findings on neurologic examination. An initial diagnosis of thoracic spinal pain and sacroiliac sprain and strain was made. At approximately 7 weeks of treatment, because of the patient's persistent pain, chest magnetic resonance imaging (MRI) was ordered by the chiropractic physician.

Interventions and Outcomes: The chest MRI revealed an intradural extramedullary mass at the T5 level creating a marked compression of the spinal cord. A thoracic MRI was conducted 2 days later confirming the presence of the tumor. The patient was successfully treated with spinal surgery to remove a schwannoma.

Conclusions: Mechanical spinal pain is a common symptom in patients presenting to chiropractic offices and may present concurrent with other pathology. In the presence of unresolved persistent thoracic pain, clinicians should investigate other potential causes. (J Chiropr Med 2017;16:242-245)
\end{abstract}

Key Indexing Terms: Neurofibroma; Tumors; Spinal Cord

\section{INTRODUCTION}

Primary spinal tumors are rare, with an incidence of 0.74 to 0.97 per 100000 person-years, and account for approximately $4 \%$ to $16 \%$ of all central nervous system tumors. $^{1-3}$ The most common sites are the spinal cord $(60.5 \%)$, followed by the spinal meninges $(36.0 \%)$, and the caudal equina $(3.5 \%) .{ }^{1}$ Primary spinal tumors are divided into 3 categories based on their anatomic location: intramedullary, intradural extramedullary, and extradural. ${ }^{4}$ Intradural extramedullary (IE) tumors include schwannomas, neurofibromas, and meningiomas. ${ }^{5}$ Schwannomas can present with 2 main symptoms - radiculopathy and neurogenic claudication - as well as worsening sensorimotor loss

\footnotetext{
${ }^{a}$ Fort Lee Chiropractic Associates, Fort Lee, New Jersey.

b Private Practice, San Diego, California.

Corresponding author: Andrew M. Rodgers, DC, MS, BS, 530 Main Street Suite 2, Fort Lee, NJ 07024. Tel.: +1 2015926200. (e-mail: chiroexcel@verizon.net).

Paper submitted June 7, 2016; in revised form January 30, 2017; accepted February 2, 2017.

1556-3707

(C) 2017 National University of Health Sciences.

http://dx.doi.org/10.1016/j.jcm.2017.02.002
}

and back pain radiating from the tumor level. ${ }^{6}$ Patients with unilateral localized spinal tumors are more likely to experience localized back pain, whereas concentric growths are associated with diffused pain and motor deficits. ${ }^{7}$

The prevalence of chronic back pain is rising and accounts for $10 \%$ of the population, with $84 \%$ of them seeking care from a health care provider. ${ }^{8}$ One of the recommended treatment options for low back pain (LBP) is spinal manipulative therapy. ${ }^{9,10}$ Chiropractors are one of the most common health care providers that perform spinal manipulation therapy for back pain. ${ }^{11}$

The purpose of this case report is to describe a patient who presented for chiropractic care for back pain and was later diagnosed with a thoracic tumor.

\section{CASE RePORT}

A 45-year-old male presented to a chiropractic practice for the first time with acute exacerbation of chronic LBP with sciatica and acute mid-back pain. Approximately 1 year before presentation, the patient had injured his lower back stepping off a curb while jogging, which had resulted in sciatic pain. An orthopedist had ordered lumbar magnetic resonance imaging 
(MRI), which revealed a small right disk extrusion at L4/L5 compressing on the descending nerve root. The patient started a regimen of physical therapy treatments, ending with 2 steroid injections into the lower back 8 months after initial injury, but the sciatic pain was not resolved.

A month later, the patient presented to a local hospital with severe rib pain and reported that the mode of injury was his child jumping on his back. Chest and thoracic radiographs were ordered, and rib fracture was ruled out. Two months later, he presented to the emergency department (ED) with severe pain in his ribs and mid-back, but he was released without any images taken and was told that his injury would take time to heal.

Approximately 2 days after the ED visit, the patient presented for chiropractic care for his back pain. He reported right-sided pain in the mid-back and along the ribs more than $50 \%$ of the time, with a pain intensity of 4 out of 10 on a numerical pain scale; the pain was provoked by twisting of the spine. He also reported having chronic lower back and sciatic pain. The patient reported that nothing seemed to alleviate his mid-back rib and lower back pain.

Examinations with positive findings included the Goldthwait test, demonstrating local pain on the right side; the Minor sign test, demonstrating sacroiliac pain; and the Yeoman test on the right, demonstrating local pain; however, the Valsalva test result was negative. Upper and lower extremity muscle strength and Achilles and patellar reflexes were normal. The patient reported no respiratory illnesses within the prior 6 months. Palpation revealed taut muscles and tenderness to the right lumbar extensors, thoracic extensors, gluteus medius and minimus, hamstrings, and piriformis. Ranges of motion of the lower spinal region were limited in extension, flexion, bilateral flexion, and rotation, accompanied by pain throughout all ranges of motion. Ranges of motion of the thoracic spine were within normal limits. On the basis of this information, the initial diagnosis was sacroiliac sprain and strain and thoracic spinal pain, and the patient's prognosis for recovery was good.

\section{INTERVENTION AND OUTCOME}

A short trial of conservative chiropractic care, including chiropractic manipulative therapy (CMT) to the sacroiliac joints and to the thoracolumbar spine, was recommended. The CMT consisted of diversified techniques, administered with the patient in the prone position; a high-velocity, low-amplitude force was delivered either by an Activator instrument (not according to Activator protocol; Activator Methods, Phoenix, AZ) or by hand directed to his mid-thoracic and side posture for his lumbar spine.

During re-examination after 3 weeks and 8 treatment visits, the patient reported pain down his leg, mid-back rib pain, and lower back pain. The patient reported that all 3 symptoms were improving with the chiropractic treatments. After the re-evaluation, the assessment was that the condition was improving as expected. Along with chiro- practic treatments, McKenzie back exercises were also initiated at this visit.

At 4 weeks of treatment, the patient returned to the ED with severe pain in the chest and ribs. A chest computed tomography (CT) scan showed negative findings. The next day, ultrasonography was ordered for his abdomen, and findings were negative again.

The patient continued with his chiropractic visits. After 7 weeks and 20 treatment visits, pain in the right-sided rib region persisted, but the pain in the lumbar spine and the sciatica continued to improve. Because of the persistent rib pain, the chiropractor referred the patient for MRI of the chest. The MRI report revealed a " $2.2 \times 1.5 \mathrm{~cm}$ mass within the central spinal canal in the mid- to upper thoracic region," and MRI of the thoracic spine was then recommended. The MRI report was as follows: "An intradural extramedullary mass at the T5 level creating marked compression of the spinal cord," and a surgical consult was recommended. The chiropractor explained the MRI reports along with the MRI (Figs 1 and 2) to the patient and immediately referred the patient to a neurosurgeon.

Four days later, the patient underwent surgery to remove the spinal tumor, and the preoperative and postoperative diagnosis was IE spinal tumor (schwannoma) and thoracic stenosis. A laminectomy with allography was performed from T4 to T6 for the thoracic stenosis. On that same day, the patient also underwent an L4 and L5 partial right laminotomy and discectomy for the L4/L5 disk extrusion.

The patient followed up with the neurosurgeon for periodic MRI checkups. At his 2-year postoperative follow-up, all examinations by MRI showed absence of any residual tumor or regrowth and no cord pressure. The patient has received physical therapy treatments to regain muscle strength and for the most part remains asymptomatic. The patient gave consent for his information to be presented in this case report.

\section{DISCUSSION}

This case is unusual in that there were several complicating factors in the clinical presentation. The patient had a history of trauma occurring close to the time of onset of the back pain, which led his health care providers to think that the pain was caused by musculoskeletal injuries. The initial examination by his chiropractor supported a diagnosis of thoracic spinal misalignment, with pinching of the local thoracic nerves and pain radiating to the intercostal nerves. Although spinal tumors are rare, they are encountered by health care practitioners and mimic mechanical spinal pain.

Other case studies have reported similar findings. Cox et al. ${ }^{12}$ reported the case of a 30-year-old man presenting with lower right back and abdominal pain provoked by a jarring motion. Magnetic resonance imaging was ordered, and an IE tumor, specifically a schwannoma at the T10 vertebral level, was discovered. The patient underwent full 


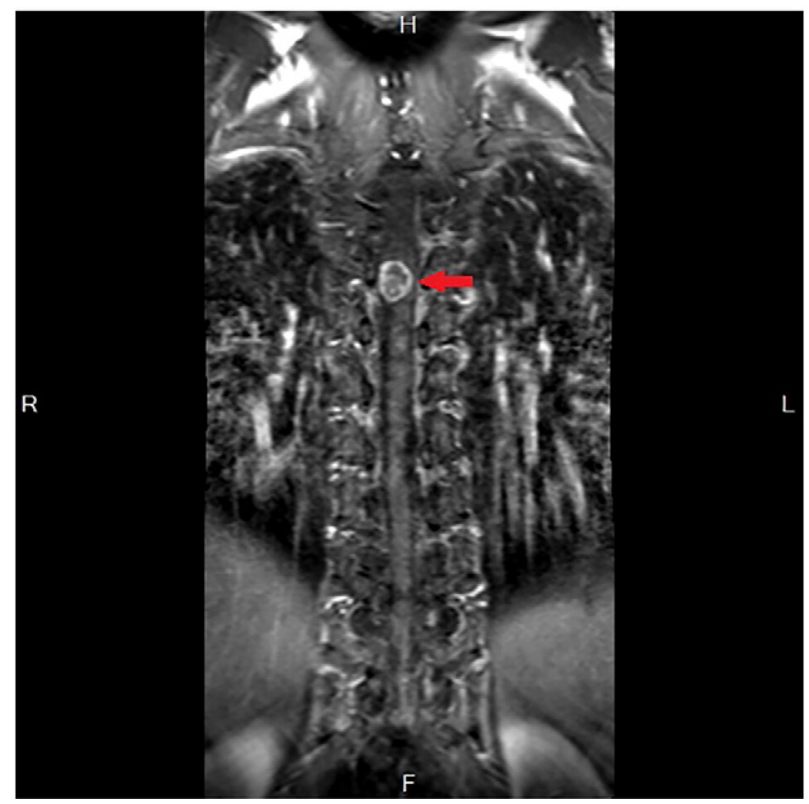

Fig I. Chest magnetic resonance imaging revealing intradural extramedullary tumor at T5 (arrow).

laminectomy of T10 and partial laminectomies of T9 and $\mathrm{T} 11$ to remove the tumor. The authors concluded that spinal tumors should be considered when undiagnosed abdominal pain is present.

Petersen et al. ${ }^{13}$ presented a case report on a 48-year-old male with recurrent acute LBP, and an MRI revealed a spinal myxopapillary ependymoma. The authors concluded that since spinal intramedullary ependymomas mimic LBP symptoms, clinicians were encouraged to be sensitive to subtle changes in the clinical presentation of LBP.

Osterhouse et al. ${ }^{14}$ discussed the case of a 25 -year-old female patient suffering from chronic mid-thoracic pain with chest radiographs revealing a "well-defined opacity." Computed tomography revealed a mass, and a CT-guided biopsy of the mass resulted in a diagnosis of a ganglioneuroma. The mass was surgically removed.

Since chiropractors commonly treat thoracolumbar spine pain, it is important for the clinician to examine other causes of persistent spine pain after a short trial of chiropractic treatments.

\section{Limitations}

As this is a case report, the findings in this study cannot be generalized to other patients with similar presentations.

\section{CONCLUSIONS}

This case report describes a 45-year-old man with a schwannoma and concurrent mechanical back pain presenting to a chiropractic office. Persistent severe back pain

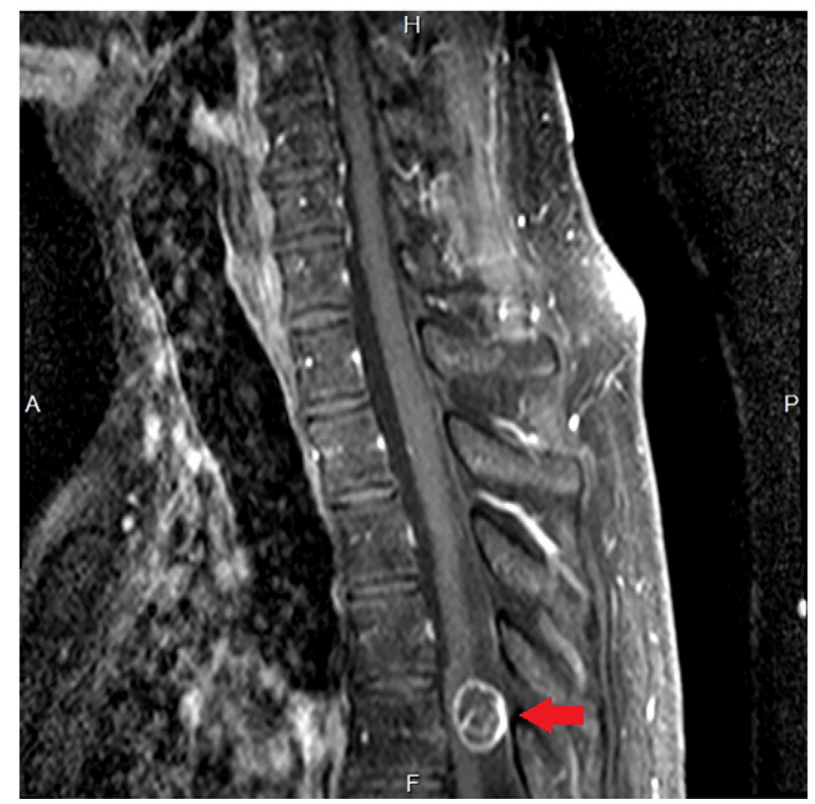

Fig 2. Thoracic magnetic resonance imaging revealing intradural extramedullary tumor at T5 (arrow).

not responding to short-term trial of chiropractic treatments should alert the clinician of other causes, and further assessment is indicated.

\section{Funding Sources and Conflicts of Interest}

No funding sources or conflicts of interest were reported for this study.

\section{CONTRIBUTORSHIP INFORMATION}

Concept development (provided idea for the research): A.M.R.

Design (planned the methods to generate the results): A.M.R.

Supervision (provided oversight, responsible for organization and implementation, writing of the manuscript): A.M.R.

Data collection/processing (responsible for experiments, patient management, organization, or reporting data): A.M.R.

Analysis/interpretation (responsible for statistical analysis, evaluation, and presentation of the results): A.M.R.

Literature search (performed the literature search): K.B.K.

Writing (responsible for writing a substantive part of the manuscript): A.M.R., K.B.K.

Critical review (revised manuscript for intellectual content, this does not relate to spelling and grammar checking): A.M.R., K.B.K. 


\section{Practical Applications}

- Although spinal tumors are rare, thoracic Schwannomas may mimic mechanical back pain presenting to chiropractic offices.

- Persistent pain not resolving after a 7-week trial of chiropractic treatments should alert the clinician to investigate other causes.

- An appropriate MRI consult for the correct region was instrumental for the diagnosis of thoracic Schwannoma in this patient.

\section{REFERENCES}

1. Duong LM, McCarthy BJ, McLendon RE, et al. Descriptive epidemiology of malignant and nonmalignant primary spinal cord, spinal meninges, and cauda equina tumors, United States, 2004-2007. Cancer. 2012;118(17): 4220-4227.

2. Schellinger KA, Propp JM, Villano JL, McCarthy BJ. Descriptive epidemiology of primary spinal cord tumors. J Neuro-Oncol. 2008;87(2):173-179.

3. Hirano K, Imagama S, Sato K, et al. Primary spinal cord tumors: review of 678 surgically treated patients in Japan. A multicenter study. Eur Spine J. 2012;21(10):2019-2026.

4. Elsberg CA. Some aspects of the diagnosis and surgical treatment of tumors of the spinal cord: with a study of the end results in a series of 119 operations. Ann Surg. 1925;81(6):1057-1073.
5. Brown KM, Dean A, Sharr MM. Thoracic intramedullary schwannoma. Neuropathol Appl Neurobiol. 2002;28(5): 421-424.

6. Kothbauer KF. Neurosurgical management of intramedullary spinal cord tumors in children. Pediatr Neurosurg. 2007; 43(3):222-235.

7. Mohme M, Mende KC, Krätzig T, et al. Impact of spinal cord compression from intradural and epidural spinal tumors on perioperative symptoms-implications for surgical decision making. Neurosurg Rev. 2016 [Epub ahead of print].

8. Freburger JK, Holmes GM, Agans RP, et al. The rising prevalence of chronic low back pain. Arch Intern Med. 2009; 169(3):251-258

9. Haas M, Vavrek D, Peterson D, Polissar N, Neradilek MB. Dose-response and efficacy of spinal manipulation for care of chronic low back pain: a randomized controlled trial. Spine J. 2014;14(7):1106-1116.

10. Chou R, Qaseem A, Snow V, et al. Clinical Efficacy Assessment Subcommittee of the American College of Physicians, American College of Physicians, American Pain Society Low Back Pain Guidelines Panel. Diagnosis and treatment of low back pain: a joint clinical practice guideline from the American College of Physicians and the American Pain Society. Ann Intern Med. 2007;147(7): 478-491 Erratum in: Ann Intern Med 2008;148(3):247248.

11. Davis MA, Sirovich BE, Weeks WB. Utilization and expenditures on chiropractic care in the United States from 1997 to 2006. Health Serv Res. 2010;45(3):748-761.

12. Cox JM, Alter M. Schwannoma: challenging diagnosis. J Manipulative Physiol Ther. 2001;24(8):526-528.

13. Petersen D, Lystad RP. Spinal myxopapillary ependymoma in an adult male presenting with recurrent acute low back pain: a case report. Chiropr Man Therap. 2016;24:11.

14. Osterhouse MD, Kettner NW, Birrer PT, Mankamyer K. Ganglioneuroma masquerading as spinal pain. J Manipulative Physiol Ther. 2002;25(3):184-187. 